International Journal of Modern Physics E (C) World Scientific Publishing Company

\title{
A Binary Scenario for the Formation of Strongly Magnetized White Dwarfs
}

\author{
J. NORDHAUS* \\ Center for Computational Relativity and Gravitation \\ Rochester Institute of Technology \\ Rochester, NY 14623, USA \\ E3 \\ National Technical Institute for the Deaf \\ Rochester Institute of Technology \\ Rochester, NY 14623, USA \\ E3 \\ Dept. of Astrophysical Sciences \\ Princeton University \\ Princeton, NJ 08544, USA \\ Received (received date) \\ Revised (revised date)
}

\begin{abstract}
Since their initial discovery, the origin of isolated white dwarfs (WDs) with magnetic fields in excess of $\sim 1 \mathrm{MG}$ has remained a mystery. Recently, the formation of these high-field magnetic WDs has been observationally linked to strong binary interactions incurred during post-main-sequence evolution. Planetary, brown dwarf or stellar companions located within a few $\mathrm{AU}$ of main-sequence stars may become engulfed during the primary's expansion off the main sequence. Sufficiently low-mass companions inspiral inside a common envelope until they are tidally shredded near the natal white dwarf. Formation of an accretion disk from the disrupted companion provides a source of turbulence and shear which act to amplify magnetic fields and transport them to the WD surface. We show that these disk-generated fields explain the observed range of magnetic field strengths for isolated, high-field magnetic WDs. Additionally, we discuss a high-mass binary analogue which generates a strongly-magnetized WD core inside a pre-collapse, massive star. Subsequent core-collapse to a neutron star may produce a magnetar.
\end{abstract}

\section{Observational Constraints}

Approximately $10 \%$ of isolated white dwarfs form with magnetic field strengths in excess of $10^{6} \mathrm{G}$. Based on Zeeman measurements, surface field strengths for these high-field magnetic white dwarfs (HFMWDs) range from a few to slightly less than a thousand megagauss $(\mathrm{MG})^{2|3| 4}$. This is in contrast to the bulk of isolated

*NSF Astronomy and Astrophysics Postdoctoral Fellow

${ }^{\dagger}$ nordhaus@astro.rit.edu 
white dwarfs with measured weak fields or non-detection upper limits of typically

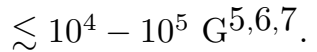

If the magnetic field strengths of white dwarfs were independent of binary interactions, then the observed distribution of isolated WDs should be similar to those in detached binaries. Remarkably, not a single detached, binary system in which the primary is a WD and the companion is an M-dwarf contains a HFMWD $8 / 9[10$. Within 20 pc, there are 109 known WDs (21 of which have a non-degenerate companion). SDSS has identified 149 HFMWDs (none of which has a non-degenerate companion). The maximum probability of obtaining samples at least this different from the same underlying population, assuming binomial statistics 11 , is $5.7 \times 10^{-10}$. This suggests at the $6.2-\sigma$ level that the two populations are different. Furthermore, SDSS identified $1253 \mathrm{WD}+\mathrm{M}$-dwarf binaries (none of which are magnetic). As was previously suggested, the presence or absence of binarity is crucial in influencing whether a HFMWD results 12 . These results initially seem to indicate that HFMWDs preferentially form when isolated. However, unless there is a mechanism by which very distant companions prevent the formation of a strong magnetic field, a more natural explanation is that highly-magnetized white dwarfs became that way by engulfing (and removing) their companions 13 .

\section{The Binary Scenario}

In the binary scenario, the progenitors of HFMWDs are those systems that incur a common envelope (CE) phase during post-MS evolution. Magnetic CVs may be the progeny of common envelope systems that almost merge but eject the envelope, leaving a close binary. Isolated HFMWDs may result from systems with less massive companions - as suggested by recent observations. In this case, the companion can't eject the envelope and thus, continues to in-spiral until it tidally disrupts. The formation of an accretion disk can both amplify magnetic fields and transport them to the WD surface. Recently, this scenario was suggested and quantitatively investigated as a way to produce an isolated HFMWD 13 . Figure 1 sketches the evolutionary pathway to forming an isolated HFMWD. A close, M-dwarf+main-sequence binary will enter a common envelope when the primary leaves the main-sequence either through direct expansion or tidal engulfment 19 . The M-dwarf continues to inspiral toward the natal WD core until it tidally disrupts and forms an accretion disk. The magnetic field is then amplified in the accretion disk and transported onto the WD surface. Mass-loss proceeds during the post-main-sequence-giant phases until the natal, magnetized WD is exposed.

In this proceeding, we highlight recent results from this scenarid 13 and show that for a range of disrupted-companion masses, the fields generated in the disk are sufficient to explain the observed range of HFMWDs. Additionally, we discuss the possible evolutionary relationships that HFMWDs have with related objects such as post-AGB stars ${ }^{14 \mid 15}$ and planetary nebulae $(\mathrm{PNe})^{16|17| 18 \mid 19}$. Finally, we comment on high-mass stellar analogues 13 . 

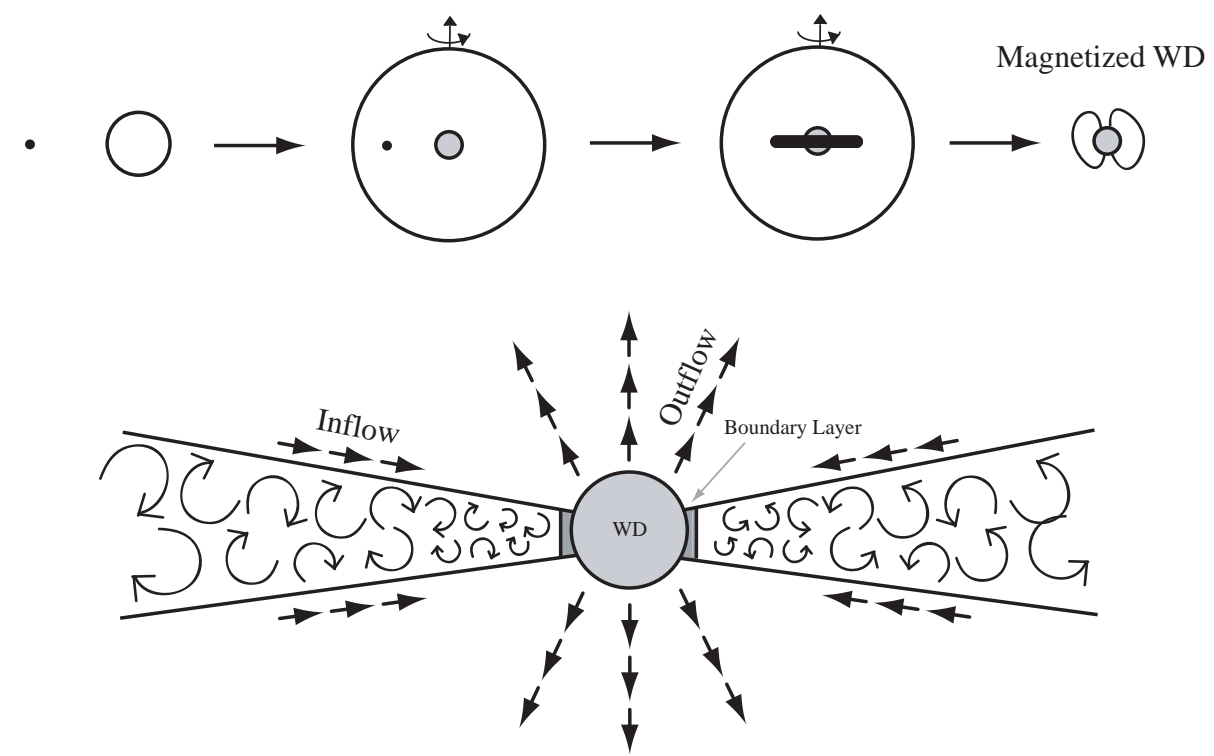

Fig. 1. A path to form an isolated, highly magnetized WD. The top panel shows an evolutionary sequence. From left to right: A close, main-sequence binary $\longrightarrow$ common envelope phase during the post-main sequence $\longrightarrow$ tidal disruption and formation of an accretion disk $\longrightarrow$ emergence of a HFMWD. Note that although the cartoon depicts a dipole field, the final magnetic geometry (small scale field vs. large scale field) will depend on details of the amplification process. The bottom panel shows the turbulent accretion disk. A conveyor belt of magnetized material flows in. Some material is lost to outflows while some is incorporated into the white dwarf. Additional field amplification may occur in the boundary layer between the slowly rotating WD and Keplerian accretion disk.

\subsection{Disk Formation}

Disks can form inside common envelopes for a wide range of companions. Jupitermass planets20, brown dwarfs and low-mass stars will tidally disrupt inside an AGB star when the self gravity of the companion is overcome by the differential gravitational force of the white dwarf core. This occurs at the tidal shredding radius, which we estimate as

$$
R_{\mathrm{s}} \simeq R_{\mathrm{c}}\left(2 M / M_{\mathrm{c}}\right)^{1 / 3},
$$

where $r_{\mathrm{c}}$ and $M_{\mathrm{c}}$ are the radius and mass of the companion and $M$ is the stellar mass interior to $R_{\mathrm{s}} 19$. Note that planetary and low-mass stellar companions to solar-type stars are significantly more plentiful than brown dwarfs 21 . Therefore, disks in the lower and higher mass ranges may be more common than those in the intermediate mass range.

Once the disk forms, it is ionized and thus, susceptible to the development of magnetized turbulence (e.g. via the magneto-rotational instability). Accretion proceeds on a viscous timescale given by $t_{\mathrm{visc}} \simeq R^{2} / \nu \simeq P_{\mathrm{orb}} / \alpha_{\mathrm{ss}}(H / R)^{2}$, where $P_{\text {orb }}$ 
is the Keplerian orbital period at radius $R, \nu$ is the effective kinematic viscosity, $H$ is the disk scaleheight, $c_{\mathrm{s}}=H \Omega$ is the midplane soundspeed, $\Omega=2 \pi / P_{\text {orb }}$ is the Keplerian orbital frequency, and $\alpha_{\mathrm{ss}}$ is the dimensionsless Shakura-Sunyaev parameter that characterizes the efficiency of angular momentum transport. Initially, the accretion rate can be approximated as

$$
\begin{aligned}
\dot{M} \sim & \frac{M_{\mathrm{c}}}{\left.t_{\mathrm{visc}}\right|_{\mathrm{s}}} \approx 7 M_{\odot} \mathrm{yr}^{-1} \times \\
& \left(\frac{\alpha_{\mathrm{ss}}}{10^{-2}}\right)\left(\frac{M_{\mathrm{c}}}{30 M_{\mathrm{J}}}\right)^{3 / 2}\left(\frac{r_{\mathrm{c}}}{r_{\mathrm{J}}}\right)^{-1 / 2}\left(\frac{H / R}{0.5}\right)^{2},
\end{aligned}
$$

where $r_{\mathrm{c}}$ is scaled to the radius of Jupiter and $H / R$ to a large value $\sim 0.5$ because the disk cannot is geometrically thick and cannot cool efficiently at high accretion rates. Additionally, since the companions under consideration lead to disks much more dense than the stellar envelope, we ignore any interaction between the disk and the star.

Equation (2) illustrates that for $M_{\mathrm{c}} \sim 0.1-500 M_{\mathrm{J}}$, and for typical values of $\alpha_{\mathrm{ss}} \sim 0.01-0.1, \dot{M}$ is initially a few to many orders of magnitude larger than the Eddington accretion rate of the proto-WD (i.e., $\dot{M}_{\text {Edd }} \sim 10^{-5} M_{\odot} \mathrm{yr}^{-1}$ ). At first, it might seem apparent that inflow of mass onto the WD surface would be inhibited by radiation pressure. However, this neglects the fact that, at sufficiently high accretion rates, photons are trapped and advected to small radii faster than they can diffuse out 22|23|24. In this 'hyper-critical' regime, accretion is possible when $\dot{M} \gg \dot{M}_{\text {Edd }}$.

Hyper-critical accretion may occur if the accretion timescale, $t_{\mathrm{visc}}$, is less than the timescale for photons to diffuse out of the disk midplane, $t_{\text {diff }} 23$. The location in the disk where this occurs is called the "trapping radius" and is given by $R_{\mathrm{tr}}=$ $\dot{M} \kappa H / 4 \pi R c$ where $\kappa$ is the opacity. An important quantity is the ratio of the trapping radius to the outer disk radius $R_{\mathrm{s}}$ (coincident with the tidal shredding radius) :

$$
\begin{aligned}
\frac{R_{\mathrm{tr}}}{R_{\mathrm{S}}} \approx & 1.0 \times 10^{4}\left(\frac{\alpha_{\mathrm{ss}}}{10^{-2}}\right)\left(\frac{\kappa}{\kappa_{\mathrm{es}}}\right) \times \\
& \left(\frac{M_{\mathrm{c}}}{30 M_{\mathrm{J}}}\right)^{11 / 6}\left(\frac{M_{\mathrm{WD}}}{0.6 M_{\odot}}\right)^{-1 / 3}\left(\frac{r_{\mathrm{c}}}{r_{\mathrm{J}}}\right)^{-3 / 2}\left(\frac{H / R}{0.5}\right)^{3},
\end{aligned}
$$

where we have used equation (2) and scaled $\kappa$ to the electron scattering opacity $\kappa_{\mathrm{es}}=0.4 \mathrm{~cm}^{2} \mathrm{~g}^{-1}$. If $R_{\mathrm{s}}<R_{\mathrm{tr}}$, then $t_{\mathrm{visc}}<t_{\mathrm{diff}}$ and photons are advected with the matter.

From equation (3) we conclude that $R_{\mathrm{tr}}>R_{\mathrm{s}}$ for $M_{\mathrm{c}} \gtrsim 0.1 M_{\mathrm{J}}$. This implies that photon pressure will be unable to halt accretion initially and that the local energy released by accretion must be removed by advection 25 . Advection acts like a conveyor belt, nominally carrying the gas to small radii as its angular momentum is removed. If there is no sink for the hot gas, this conveyor may "jam". This is an important distinction between white dwarfs and neutron stars or black holes, 
as the latter two can remove the thermal energy by neutrino cooling or advection through the event horizon, respectively. For WDs, neutrino cooling is ineffective and thermal pressure builds, such that radiation pressure may again become dynamically significant. However, this scenario neglects the possibility of outflows, which can sustain inflow by carrying away the majority of the thermal energy, thereby allowing a smaller fraction of the material to accrete at a higher than Eddington rate. Though more work is needed to assess the efficiency of outflows in the present context, radiatively-inefficient accretion flows are widely thought to be prone to powerful outflows 26127. Even if accretion occurs at, or near, the Eddington rate, the fields produced ( $\gtrsim 10 \mathrm{MG}$ ) are still strong enough to explain the bulk of the magnetized WD population. The origin of the strongest field systems $(\sim 100-1000$ MG) may be problematic if accretion is limited to the Eddington rate.

Nevertheless, the material deposited outside the WD will be hot and virialized, forming an extended envelope with a lengthscale comparable to the radius. Though hot, this material will eventually cool on stellar timescales and become incorporated into the stellar layers near the proto-WD surface. If this material cools at some fraction of the local Eddington luminosity, it will be incorporated onto the WD surface on a timescale $\sim 10^{2}-10^{4}$ years - much less than typical AGB lifetimes.

\subsection{Disk Dynamo}

Due to the presence of shear in the disk, the MRI is a likely source of turbulence, and can supply the conditions by which magnetic field amplification can occur. Large-scale fields produced by the MRI have been modeled via $\alpha-\Omega$ dynamos at various levels of sophistication. However, for the purposes of estimating orders of magnitude of the fields, approximate values from less sophisticated treatments can be employed as we do now. The Alfvén velocity in the disk obeys $v_{\mathrm{a}}^{2}=\alpha_{\mathrm{ss}} c_{\mathrm{s}}^{2}$, such that the mean toroidal field at radius $R$ is given by 28

$$
\begin{aligned}
\bar{B}_{\phi} \sim & \left(\frac{\dot{M} \Omega}{R} \frac{R}{H}\right)^{1 / 2} \\
\approx & 160 \mathrm{MG}\left(\frac{\eta_{\mathrm{acc}}}{0.1}\right)^{1 / 2}\left(\frac{\alpha_{\mathrm{ss}}}{10^{-2}}\right)^{1 / 2}\left(\frac{\mathrm{M}_{\mathrm{c}}}{30 \mathrm{M}_{\mathrm{J}}}\right)^{3 / 4} \times \\
& \left(\frac{M_{\mathrm{WD}}}{0.6 M_{\odot}}\right)^{1 / 4}\left(\frac{r_{\mathrm{c}}}{r_{\mathrm{J}}}\right)^{-1 / 4}\left(\frac{H / R}{0.5}\right)^{1 / 2}\left(\frac{R}{10^{9} \mathrm{~cm}}\right)^{-3 / 4},
\end{aligned}
$$

where in the second equality we have substituted equation (2) for $\dot{M}$, and multiplied by the factor $0.1 \lesssim \eta_{\text {acc }} \leq 1$ to account for the possibility of outflows as described above. If an $\alpha-\Omega$ dynamo operates, the mean poloidal field $\bar{B}_{p}$ is related to the toroidal field via $\bar{B}_{p}=\alpha_{\mathrm{ss}}^{1 / 2} \bar{B}_{\phi} 28$. However, regardless of whether a large scale field is generated, a turbulent field of magnitude $\bar{B}_{\phi}$ is likely to be present and contributing significantly to the Maxwell stresses responsible for disk accretion. 
The toroidal field evaluated near the WD surface $R \approx R_{\mathrm{WD}} \approx 10^{9} \mathrm{~cm}$ as a function of companion mass $M_{\mathrm{c}}$, calculated for two different values of the viscosity $\left(\alpha_{\mathrm{ss}}=0.01\right.$ and 0.1$)$ is shown in Fig. 2 . In both cases, we assume that $\eta_{\mathrm{acc}}=0.1$.

Note that for the range of relevant companion masses $M_{\mathrm{c}} \sim 0.1-500 M_{\mathrm{J}}$, $\bar{B}_{\phi} \sim 10-1000 M G$, in precisely the correct range to explain the inferred surface field strengths of HFMWDs (see Fig. 2 below).

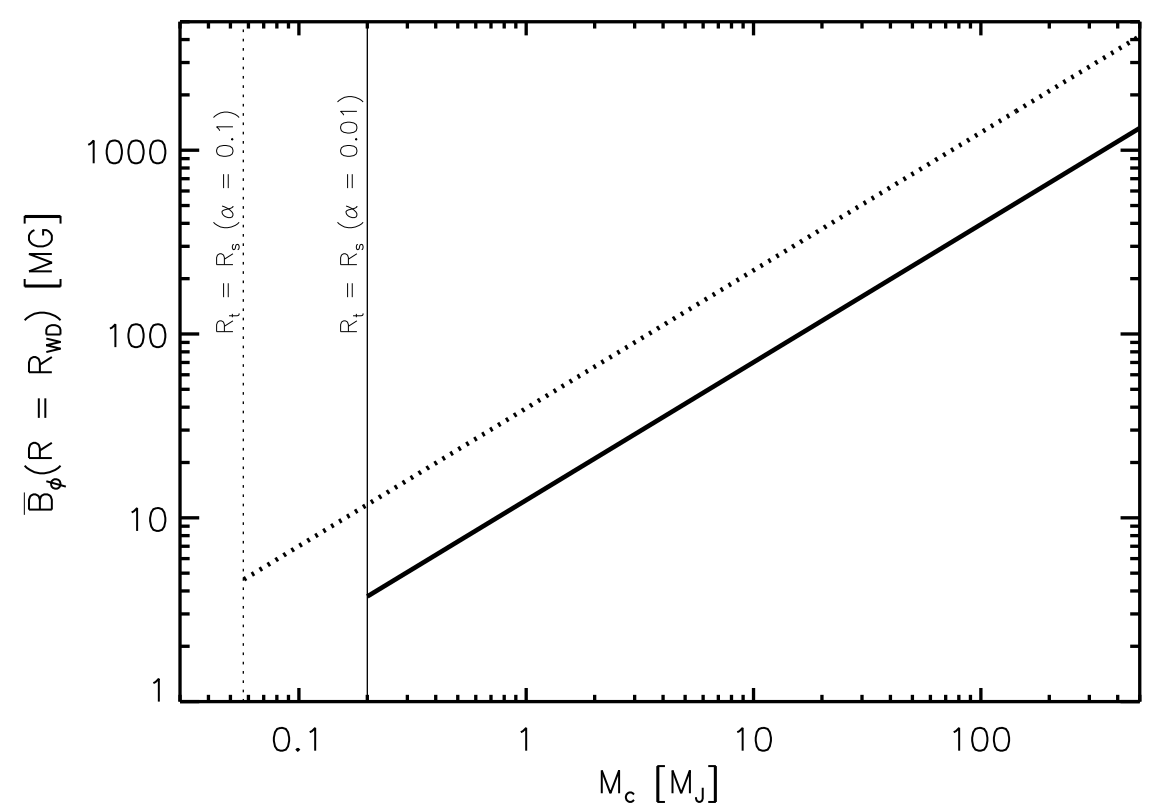

Fig. 2. Toroidal magnetic field strength, $\bar{B}_{\phi}$ (eq. [4), at the WD surface as a function of the mass of the tidally disrupted companion $M_{\mathrm{c}}$. Toroidal field strengths are presented for two values of the viscosity, $\alpha_{\mathrm{ss}}=0.01$ (solid line) and $\alpha_{\mathrm{ss}}=0.1$ (dotted line) and assuming an accretion efficiency $\eta_{\mathrm{acc}}=0.1$. The white dwarf mass and radius are $M_{\mathrm{WD}}=0.6 M_{\odot}$ and $r_{\mathrm{WD}}=10^{9} \mathrm{~cm}$, respectively. The vertical lines show the companion mass above which photons are trapped in the accretion flow (i.e., $r_{\mathrm{tr}}>r_{\mathrm{s}}$; eq. [3), such that super-Eddington accretion occurs.

\section{Proto-Planetary and Bipolar Planetary Nebulae}

If the origin of highly magnetized WDs lie in common envelope phases, then they may also exist around proto-planetary (PPNe) and planetary nebulae (PNe). For these systems, the link between close binary interactions and the formation of $\mathrm{PPNe} / \mathrm{PNe}$ has been well established 14|15|29]. It's interesting to also note that the presence of outflows inside the AGB star, near the proto-WD surface is al- 
ready coincident with the prevalence of outflows in proto-planetary nebulae. The direct detection of a HFMWD in a PPN or PN system would provide additional evidence for the binary scenario. Recent, indirect evidence in the form of hot Xray emitting gas in two post-CE PN systems has been discovered and could be due to magnetic activity or accretion onto the WD 30 . Additionally, observations of masers in post-AGB water fountain sources show magnetically-shaped, bipolar jet-like structures $31 \mid 32$.

\section{Higher Mass Stellar Analogues and Magnetars}

While $\sim 10 \%$ of WDs are highly magnetized, it's interesting to note that $\sim 10 \%$ of neutron stars are highly magnetized. High-mass stars may also undergo common envelope interactions in the presence of close companions. If the common envelope field mechanisms described in this proceeding and in our previous paper 13 operate in high-mass stars, then the result could be strong field neutron stars or magnetars (neutron stars with magnetic fields in excess of $\sim 10^{14}-10^{15} \mathrm{G}$ ). In particular, formation of an accretion disk from an engulfed companion during a red supergiant phase could produce a magnetized WD core. In the eventual core-collapse and stellar supernova explosion (possibly driven by the neutrino mechanism; 33134 ), the magnetized WD core collapses to a neutron star. If simple flux freezing operates (itself an open question) and the initial magnetized core is on the order of $\sim 100$ $1000 \mathrm{MG}$, homologous collapse to a neutron star would generate $\sim 10^{14}-10^{15} \mathrm{G}$ fields. Typical neutron stars that possess modest field strengths may originate from core-collapse supernova of single stars or stars without having incurred a CE phase. Note that what we are proposing is an alternative to the neutrino-driven convection dynamo 35 . In our scenario, the engulfment of a companion and the formation of an accretion disk naturally provides fast rotation, magnetized turbulence and differential rotation - the same ingredients that are also needed for the neutrino-driven convection dynamo.

\section{Conclusions}

In summary, common envelope evolution as the origin of highly-magnetized, white dwarfs is strongly supported by observations. For HFMWDs, whether the scenario described in this proceeding and our previous paper ${ }^{13}$ is ultimately found to be

viable will depend on the statistics of low-mass stellar and substellar companions 36 to stars of similar masses to (or somewhat higher masses than) the Sun. The numerous radial velocity searches of the last 20 years have revealed a number of such companions though the precise occurrence rate of companions in orbits that could lead to the kind of disk-dynamo mechanism remains unclear. Further theoretical work into the binary origin of HFMWDs and magnetars requires the development of multi-dimensional, magnetohydrodynamic simulations of the CE phase. 


\section{Acknowledgements}

JN is supported by an NSF Astronomy and Astrophysics Postdoctoral Fellowship under award AST-1102738 and by NASA HST grant AR-12146.01-A.

\section{References}

1. J. Liebert, P. Bergeron, \& J. B. Hoberg Astronomical Journal 125 (2003) 348.

2. B. T. Gansicke, F. Euchner, \& S. Jordan Astronomy and Astrophysics 394 (2002) 957.

3. G. D. Schmidt et al. Astrophysical Journal 595 (2003) 1101.

4. K. M. Vanlandingham et al. Astronomical Journal 130 (2005) 734.

5. A. Kawka, S. Vennes, G. D. Schmidt, D. T. Wickramasinghe \& R. Koch Astrophysical Journal 654 (2007) 499

6. G. Valyavin et al. Astrophysical Journal 648 (2006) 559.

7. R. Aznar Cuadrado et al. Astronomy and Astrophysics 423 (2004) 1081.

8. J. Liebert et al. Astronomical Journal 129 (2005) 2376.

9. N. M. Silvestri et al. Astronomical Journal 131 (2006) 1674.

10. N. M. Silvestri et al. Astronomical Journal 134 (2007) 741.

11. D. S. Spiegel, F. Paerels \& C. A. Scharf Astrophysical Journal 658 (2007) 288.

12. C. A. Tout et al. MNRAS 387 (2008) 897.

13. J. Nordhaus, S. Wellons, D. S. Spiegel, B. D. Metzger, E. G. Blackman Proceedings of the National Academy of Science 108 (2011) 3135.

14. J. Nordhaus \& E. G. Blackman MNRAS 370 (2006) 2004.

15. J. Nordhaus, E. G. Blackman \& A. Frank MNRAS 376 (2007) 599.

16. B. Miszalski et al. MNRAS 413 (2011) 1264.

17. J. Nordhaus et al. Astrophysical Journal Letters 684 (2008) L29.

18. B. Miszalski et al. Astronomy and Astrophysics Letters 488 (2008) L79.

19. J. Nordhaus et al. MNRAS 408 (2010) 631.

20. D. S. Spiegel, A. Burrows \& J. Milson Astrophysical Journal 727 (2011) 57.

21. J. Farihi, E. E. Becklin \& B. Zuckerman Astrophysical Journal Supplements 161 (2005) 394.

22. S. A. Colgate Astrophysical Journal 163 (1971) 221.

23. J. M. Blondin Astrophysical Journal 308 (1986) 755.

24. R. A. Chevalier Astrophysical Journal 346 (1989) 847.

25. H. C. Spruit The Neutron Star - Black Hole Connection Eds. C. Kouveliotou, J. Ventura, \& E. van den Heuvel (2001) 141.

26. J. Hawley \& S. A. Balbus Astrophysical Journal 573 (2002) 738.

27. K. Ohsuga, M. Mori, T. Nakamoto \& S. Mineshige Astrophysical Journal 628 (2005) 368.

28. E. Blackman, A. Frank \& C. Welch Astrophysical Journal 546 (2001) 288.

29. O. De Marco PASP $\mathbf{8 7 8}$ (2009) 316.

30. R. Montez, O. De Marco, J. H. Kastner \& Y. Chu Astrophysical Journal 721 (2010) 1820.

31. N. Amiri, W. Vlemmings, H. J. van Lagenvelde $A \xi \xi A 532$ (2011) 149.

32. W. Vlemmings \& H. J. van Lagenvelde $A \& A 488$ (2008) 619.

33. J. Nordhaus, A. Burrows, A. Almgren, J. B. Bell Astrophysical Journal 720 (2010) 694.

34. J. Nordhaus et al. Physical Review D 82 (2010) 103016.

35. R. C. Duncan \& C. Thompson Astrophysical Journal Letters 392 (1992) 9.

36. E. Bear \& N. Soker MNRAS 411 (2011) 1792. 